2005 ( $\mathrm{n}=5321), 2006$ ( $\mathrm{n}=10213), 2007$ ( $\mathrm{n}=4848))$ were visited by an interviewer then a nurse; the interview was supplemented by physical measurements using standardised protocols. Blood pressure was measured three times with an Omron HEM207 after a 5-min rest. Mean of second and third readings in participants who had not eaten, drunk alcohol, smoked, or exercised in the preceding $30 \mathrm{~min}$ were used.

Main Outcome Measures Hypertension was defined as systolic blood pressure $\geq 140 \mathrm{~mm} \mathrm{Hg}$, diastolic blood pressure $\geq 90 \mathrm{~mm} \mathrm{Hg}$, and/or taking prescribed medication to lower blood pressure.

Results A higher proportion of participants in London than elsewhere in England with survey-defined hypertension were on treatment (2005-2007 average: 61\% men, 66\% women in London; 43\% men, 55\% women in England, ( $p$ for London vs rest of England $<0.001$ for each sex). Regression analysis showed this regional effect for odds of treatment persisted after adjustment for demographic, socio-economic, and health behaviours (OR 1.48, 95\% CI 1.04 to 2.10, $\mathrm{p}=0.029$ ) and was strengthened (OR1.87 (1.25 to 2.81), $\mathrm{p}=0.003$ ) by including self-reported health, long-standing illness, diabetes, and cardiovascular disease in the model. Apart from the regional differences, treatment for hypertension increased with age and was more likely among women (OR 1.59 (1.29 to 1.97), $\mathrm{p}=0.001$ ); former smokers (OR 1.44 (1.05 to 1.99), $\mathrm{p}=0.026$ ); and people who were married; were overweight (OR 1.40 (1.03 to 1.89), $\mathrm{p}=0.033$ ) or obese (OR 1.80 (1.32 to 2.42), $\mathrm{p}<0.001)$; reported limiting (OR 2.49 (1.93 to 3.20), $\mathrm{p}<0.001$ ) or non-limiting (OR 3.25 (2.48 to 4.24), $\mathrm{p}<0.001)$ long-term illness; or reported diabetes (OR 2.36 (1.60 to 3.47$), \mathrm{p}<0.001$ ) or cardiovascular disease (OR 1.54 (1.18 to 22.02$), p=0.002)$. Treatment was $39 \%$ and $61 \%$ less likely in widowed $(p=0.004)$ and co-habiting participants $(p<0.001)$, respectively, and $40 \%$ less likely in binge-drinkers $(p=0.014)$.

Conclusion The proportion of people in London being treated for hypertension is above the national average even after adjustment for sociodemographic and health-related factors. This may be due to greater population mobility in London with more people having new Patient Health checks. Education and financial incentives for improvements in detection, treatment and control of hypertension in primary care in England have been beneficial but remain inadequate.

\section{P58 THE DEATH OF SOCIAL FRAGMENTATION: A SECONDARY ANALYSIS OF HEALTH SURVEY DATA}

doi:10.1136/jech.2010.120477.58

A Maguire, D O'Reilly. Centre for Public Health, Queen's University, Belfast, UK

Background Social Fragmentation is the idea that isolation and disorganisation within an area influences individual health. Some, but not all, studies have shown it to be related to suicide and parasuicide risk, higher GHO12 scores and higher admission rates for psychoses. The aim of this study is to determine if fragmentation per se affects mental health or if the association is due to other factors relating to fragmented areas.

Methods A measure of social fragmentation was constructed from four census variables (as per Condgon, 1996) for each of the 890 super-output areas in Northern Ireland (avg pop. 1900). These were divided into quintiles and added to the 2005 Health and Social Wellbeing Survey (HSWB) as a contextual variable. Respondent characteristics known to be associated with mental health were included such as age and sex, marital status, living alone, perceived social support, socio-economic status (based on car availability and housing tenure) and health status (based on limiting long-standing illness (LLTI)). A GHQ-12 score of 4 or more was taken as indicative of significant psychological ill health. Logistic regression analysis was restricted to 3306 individuals aged 25-74 years.
Results As expected, people in the most fragmented quintile were more likely to be unmarried and living in single person households, much more likely to be deprived, and were more likely to have a significant psychological disorder (OR 1.70, 95\% CI 1.30 to 2.24), after adjusting for age and sex. Although level of perceived social support was strongly associated with GHQ12 score, adjustment for this did not significantly explain the likelihood of poor mental health across fragmentation quintiles (OR 1.44, 95\% CI 1.08 to 1.91). However, adjustment for SES and LLTI completely eliminated the association between social fragmentation and psychological ill health. Conclusions Social Fragmentation is associated with poor mental health, but only because these areas tend to be more deprived. After adjustments are made for SES, social fragmentation has no association with the likelihood of psychological disorder. It's who you are not where you live that determines mental health. However, before we completely sound the death knoll for social fragmentation we should take into consideration the recognised imperfections of the construct and modify it. Until then, policies to improve mental health should focus on reducing individual poverty and material disadvantage rather than changing the character of areas.

\section{P59 RESIDENTIAL HISTORIES AND CONTEMPORARY MORTALITY GEOGRAPHY: USING DATA LINKAGE TO DEVELOP A DATA SET DESCRIBING MOBILITY BETWEEN BIRTH AND DEATH}

doi:10.1136/jech.2010.120477.59

${ }^{1} \mathrm{H}$ Tunstall, ${ }^{1} \mathrm{~K}$ E Pickett, ${ }^{2} \mathrm{D}$ Dorling. ${ }^{1}$ Department of Health Sciences, University of York, York, UK; ${ }^{2}$ Department of Geography, University of Sheffield, Sheffield, UK

Background and Objectives There are marked inequalities in mortality rates between areas of Britain. These inequalities have been persistent over long time periods and evaluation of recent area-based social policies in deprived areas has found that mortality rates have proven more resistance to change than other social indicators. Migration has been considered as one process that may underlie the persistence of health inequalities between areas. The geography of contemporary mortality rates is the product of movements across the life course however analysis of mobility over long time periods has been hampered by the limited availability of the necessary data in censuses, surveys and other secondary sources. The aim of this study was to assess if new detailed data sets describing residential histories between birth and death could be created through linkage of historical and contemporary data sources and used to illuminate current mortality geography.

Methods An age- and sex-structured random sample of 250 people dying in York was selected from death registrations in 2000/2001. The addresses of the deceased were traced in birth, marriage and electoral registers, BT phone books, street and trade directories and other sources. The distance between place of birth and death, time at last residence and how migration patterns varied with individual and neighbourhood characteristics were assessed within the context of data describing the health and socio-demographic history of the case study area.

Results Key residential data were successfully traced including place of birth and number of years resident at last address, collected for over $80 \%$ of cases. Only a third of those dying within York had been born in York but the majority were born in Yorkshire. Residential histories were shaped by a sub-regional network of movements linked to the development of the local economy, slum clearance and public housing policies. While the majority of the deceased in York were in-migrants half had been at their place of death for over 10 years and some for several decades. An exception to this pattern was those dying in nursing homes who often had moved shortly before death. 